\title{
Design, fabrication, and characterization of diffraction gratings for neutron phase contrast imaging
}

\author{
C. Grünzweig, ${ }^{1, a)}$ F. Pfeiffer, ${ }^{1,2}$ O. Bunk, ${ }^{1}$ T. Donath, ${ }^{1}$ G. Kühne, ${ }^{1}$ G. Frei, ${ }^{1}$ M. Dierolf, ${ }^{1}$ \\ and C. David ${ }^{1}$ \\ ${ }^{1}$ Paul Scherrer Institut, CH-5232 Villigen-PSI, Switzerland \\ ${ }^{2}$ Ecole Polytechnique Fédérale de Lausanne, CH-1015 Lausanne, Switzerland
}

(Received 13 March 2008; accepted 28 April 2008; published online 23 May 2008)

\begin{abstract}
We have developed a neutron phase contrast imaging method based on a grating interferometer setup. The principal constituents are two absorption gratings made of gadolinium and a phase modulating grating made of silicon. The design parameters of the setup, such as periodicity, structure heights of the gratings, and the distances between the gratings, are calculated. The fabrication of each grating is described in detail. The produced diffraction gratings were finally characterized within the setup, by locally evaluating the produced contrast (visibility) in each detector pixel, resulting in a visibility map over the whole grating size. An averaged value of $23 \%$ is achieved. () 2008 American Institute of Physics. [DOI: 10.1063/1.2930866]
\end{abstract}

\section{INTRODUCTION}

In quantum mechanics, light is considered to have both a wavelike and a particlelike behavior. De Broglie in 1923 extended this dualism of light to all moving massive particles. The first proof with neutrons as massive particles showing the wavelike behavior was realized within the neutron interferometry experiment in 1974 by Rauch et al. ${ }^{1}$ They used a perfect crystal interferometer measuring directly neutron phase variations. The maximum usable beam size of such a device is nowadays $10 \times 40 \mathrm{~mm}^{2} .^{2}$ Over the past years, many interesting neutron interferometry experiments have been performed with this interferometer type. ${ }^{3}$ Later on, two other techniques have been developed to detect changes in the wave front profile. The diffraction enhanced imaging (DEI) method is based on a crystal diffractometer and measures refraction effects (phase gradients) with high angular sensitivity. ${ }^{4}$ The largest beam cross section illuminating the sample using such a device is given to be $5 \times 5 \mathrm{~cm}^{2}{ }^{5}$ The other method is called free-space-propagation or in-line holography technique and delivers edge-enhanced image contrast including information on the second derivative of the phase. ${ }^{6,7}$ The sample size is therefore related to the pinhole to sample distance and ranges up to tens of millimeters in diameter.

However, some practical difficulties arise concerning the efficiency of all three techniques, besides the limited field of view. In the case of perfect crystal interferometers and crystal diffractometers, high temporal coherence (a high degree of monochromaticity) is required; hence, only a well defined wavelength of the whole spectrum is used. Both techniques are also limited by the accepted beam divergence of only a few mrad due to the use of crystal optics. The in-line holography technique is limited in efficiency since it requires high

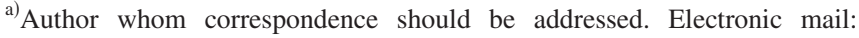
christian.gruenzweig@psi.ch.
}

spatial coherence, which can only be achieved by placing a very small aperture $\left(0.4 \mathrm{~mm}\right.$ in diameter $\left.{ }^{6}\right)$ at the beam exit port defining the incident neutron beam. These strict demands on the coherence requirements of the above mentioned techniques lead to exposure times of several hours per image even at the most intense neutron sources.

Previously, we have reported on a new neutron interferometer technique based on diffraction gratings. ${ }^{8}$ Using the grating interferometer setup, we overcome the problems limiting the other techniques concerning efficiency since the requirements for spatial and temporal coherence of the grating interferometer are very moderate. Our setup is working with a polychromatic spectrum and beam sizes of several centimeters in diameter. The efficiency of the setup allows us to record quantitative projections and even three-dimensional tomographic reconstructions of the refractive index. ${ }^{8}$ Our technique measures refraction angles $\left(\approx 10^{-6} \mathrm{rad}\right)$ similar to the DEI method. Moreover, this angle is proportional to the first derivative of the phase front profile, resulting in differential phase contrast images. An integration along the gradient direction yields the information about the phase shift. The neutron grating interferometer setup is similar to the grating based interferometer for $\mathrm{x}$ rays, which was also developed at the Paul Scherrer Institut (PSI) over the past years. $^{9-11}$

In this article, particular focus is put on the calculation of the neutron setup parameters including the grating parameters (periodicity and structure heights) and the fabrication of the gratings. The performance of the gratings is experimentally tested by measuring the resulting contrast of the grating interferometer setup, i.e., by locally evaluating the visibility over the grating area.

\section{DESIGN PARAMETERS OF THE DIFFRACTION GRATINGS AND THE SETUP}

The experimental setup consists of three gratings, as shown in Fig. 1. Figure 1(a) shows the source grating $G_{0}$ 


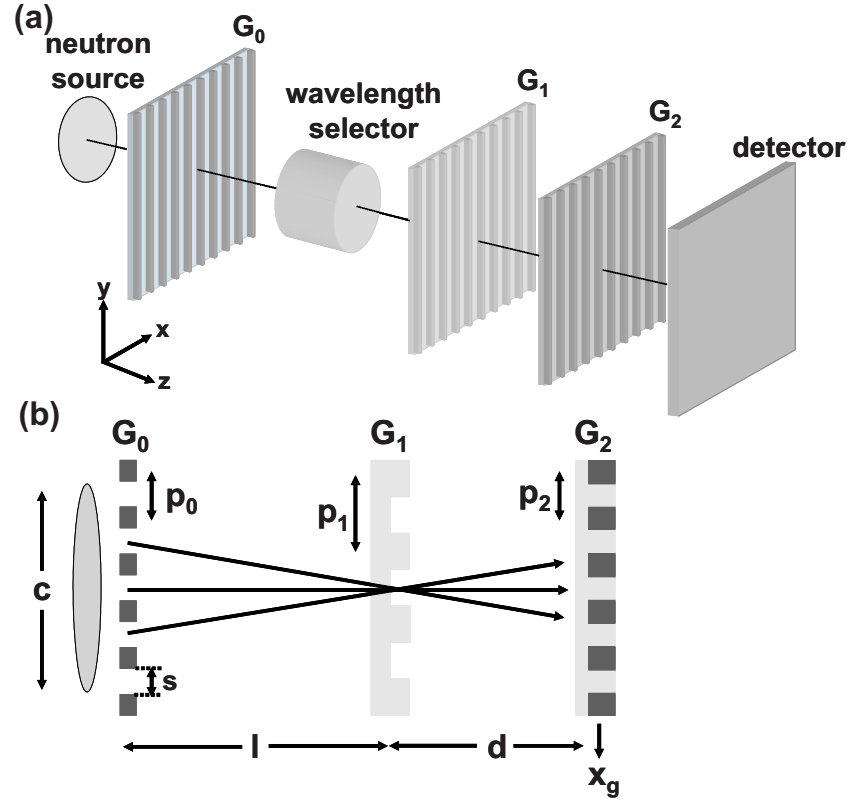

FIG. 1. (Color online) (a) Setup of the grating interferometer with the source grating $G_{0}$, phase grating $G_{1}$, and analyzer grating $G_{2}$. (b) Top view of the layout denoting the geometrical setup parameters.

consisting of absorbing gadolinium (Gd) lines placed close to the beam exit port, a beam splitter or phase grating $G_{1}$ made of silicon $(\mathrm{Si})$, and a $\mathrm{Gd}$ analyzer grating $G_{2}$. The contrast of the interferometer is formed by $G_{1}$ and $G_{2}$. The source grating $G_{0}$ allows the use of large incoherent neutron sources since it creates an array of individual line sources each providing enough spatial coherence for the interferometric contrast. The images created by each line source are superimposed congruently in the detector plane leading to a gain in intensity. The moderate temporal coherence requirements of the grating interferometer can be satisfied using a velocity selector with a bandwidth of $\pm 10 \%$ around the design wavelength $\lambda$.

For the calculation of the grating and setup parameters, some constraints are given, either by the geometry and wavelength spectrum of the beam line ${ }^{12}$ or by the feasibility of the grating fabrication processes. On one hand, this is the design wavelength $\lambda=4.1 \AA$ and the distance $l=5.23 \mathrm{~m}$ between $G_{0}$ and $G_{1}$ [see Fig. 1(b)]. On the other hand, the period $p_{2}$ of the analyzer grating $G_{2}$ [see Fig. 1(b)] was set to $p_{2}=4 \mu \mathrm{m}$ because of the limitations in the fabrication process. The other parameters in Fig. 1(b), such as the distance between $G_{1}$ and $G_{2}$ named $d$, the period $p_{0}$ of the source grating, the period $p_{1}$ of the phase grating, and the structure heights of each grating, are calculated from the given parameters $\lambda$ and $l$.

The period of the source grating is given by ${ }^{13}$

$$
p_{0}=\frac{\lambda l}{p_{2}}+\sqrt{\left(\frac{\lambda l}{p_{2}}\right)^{2}+2 \lambda l} .
$$

For the given parameters $\lambda, p_{2}$, and $l$, this results in a period of $p_{0}=1076 \mu \mathrm{m}$.

The phase grating $\left(G_{1}\right)$ acts as a beam splitter grating and divides the incoming wave front with radius $l$ [distance between $G_{0}$ and $G_{1}$, see Fig. 1(b)] essentially into the \pm 1 diffraction orders. ${ }^{14}$ The period $p_{1}$ is given by

$$
p_{1}=\frac{2 p_{0} p_{2}}{p_{0}+p_{2}},
$$

resulting in $p_{1}=7.97 \mu \mathrm{m}$ for our setup.

Through the Talbot effect, ${ }^{15}$ a linear periodic fringe pattern, which is perpendicular to the optical axis and parallel to the lines of $G_{1}$, with the period of $p_{2}$ is produced. In the plane wave case, it has its highest contrast at a distance $d_{\mathrm{pl}}$ $=p_{1}^{2} / 8 \lambda$ behind $G_{1}$. For a spherical wave, $d_{\mathrm{pl}}$ rescales to

$$
d_{\mathrm{sph}}=\frac{l d_{\mathrm{pl}}}{l-d_{\mathrm{pl}}}=\frac{l \frac{p_{1}^{2}}{8 \lambda}}{l-\frac{p_{1}^{2}}{8 \lambda}},
$$

yielding to $d_{\mathrm{sph}}=19.4 \mathrm{~mm}$ in our case.

The direct determination of the exact fringe position in the detector plane would require detectors with micrometer spatial resolution, which is not possible with neutron imaging technology. Therefore, the analyzer grating $G_{2}$, with the same periodicity $p_{2}=4 \mu \mathrm{m}$ and orientation as the fringes created by $G_{1}$, is placed at $d=d_{\text {sph }}$ [Fig. 1(b)]. $G_{2}$ transforms the fringe positions into intensity modulations on the detector located directly behind the grating.

As generally defined, the spatial coherence length $t$ is related to the size $f$ of the source and the distance $r$ to it: $t=\lambda r / f$. In our case, the spatial coherence length produced by each line source of $G_{0}$ in front of the phase grating has to be on the order of $t=4 \mu \mathrm{m}$. Thus, the size of an individual line source $s$ [see Fig. 1(a)] emitting at $\lambda=4.1 \AA$ and placed at a distance $l=5.23 \mathrm{~m}$ should be smaller than $500 \mu \mathrm{m}$. Therefore, $G_{0}$ creates an array of intrinsically coherent but mutually incoherent line sources.

The two Gd absorption gratings $G_{0}$ and $G_{2}$ should be as opaque as possible for neutrons for optimum performance of the system. For $\lambda=4.1 \AA$, the neutron $(1 / e)$ length for $\mathrm{Gd}$ is $3 \mu \mathrm{m} .{ }^{16}$ For our setup, the source grating $G_{0}$ is composed of two identical wafers each with a Gd thickness of $10 \mu \mathrm{m}$. The two wafers are stacked with the Gd layers facing each other, resulting in a total $\mathrm{Gd}$ thickness of $20 \mu \mathrm{m}$ for $G_{0}$ and an absorption of $99.9 \%$. The use of two wafers instead of one single wafer holds the advantage that one is able to adjust the slit width $s$ [see Fig. 1(b)] of the source grating and therefore manipulate the spatial coherence length produced by each individual line source.

For $G_{2}$, we were able to produce Gd lines of a thickness of up to $11 \mu \mathrm{m}$ (see next section), which also provides sufficient absorption (97\%). The height $h_{1}$ of the Si phase grating has to be such that the neutrons traversing the grating bars undergo a phase shift of $\pi$. Accordingly, $h_{1}$ is given by

$$
h_{1}=\frac{\pi}{N b_{c} \lambda}=\frac{\pi}{n_{\mathrm{SLD}} \lambda},
$$

where $N$ is the particle density and $b_{c}$ is the coherent scattering length. The product of both is known as the neutron scattering length density $\left(n_{\mathrm{SLD}}\right) .{ }^{16}$ This results in a Si structure height of $h_{1}=37 \mu \mathrm{m}$ at $4.1 \AA$. Since the Si grating lines 
TABLE I. Fabrication parameters of the diffraction gratings.

\begin{tabular}{lccc}
\hline \hline & $\begin{array}{c}\text { Source grating } \\
G_{0}\end{array}$ & $\begin{array}{c}\text { Phase grating } \\
G_{1}\end{array}$ & $\begin{array}{c}\text { Analyzer grating } \\
G_{2}\end{array}$ \\
\hline Material & $\mathrm{Gd}$ & $\mathrm{Si}$ & $\mathrm{Gd}$ \\
Period $(\mu \mathrm{m})$ & 1076 & 7.97 & 4 \\
Height $(\mu \mathrm{m})$ & $2 \times 10$ & 37 & 11 \\
Duty cycle & 0.4 & 0.5 & $\approx 0.25$ \\
\hline \hline
\end{tabular}

have negligible absorption $\left(10^{-3} \%\right), G_{1}$ can be considered as a purely phase modulating grating. An overview of all grating parameters concerning material, calculated periods, calculated structure heights, and required duty cycles (DCs, ratio of the gap to the period of the grating) is given in Table I.

\section{FABRICATION OF THE DIFFRACTION GRATINGS}

As seen from Table I, the requirements for each grating concerning the aspect ratio (ratio between structure height and structure width), material, and DC are quite different. Both the source grating $G_{0}$ and the analyzer grating $G_{2}$ have to be fabricated from Gd with a sufficient structure height to absorb the neutrons. Since the period of $G_{2}$ is by a factor of about 250 smaller than that of $G_{0}$, two different fabrication processes needed to be developed.

The source grating $G_{0}$ is an array of absorbing lines with a period of $1076 \mu \mathrm{m}$, a Gd structure height of $20 \mu \mathrm{m}$, and a DC of 0.4. The corresponding Gd linewidth has then to be fabricated to $646 \mu \mathrm{m}$. The source width $s$ for each line source is then $430 \mu \mathrm{m}$, which matches the coherence requirements of a calculated value for $s \leqslant 500 \mu \mathrm{m}$. Each wafer is fabricated on a $100 \mathrm{~mm}$ diameter quartz wafer with a thickness of $500 \mu \mathrm{m}$. The individual fabrication steps are illustrated on the top part of Fig. 2. A $20 \mathrm{~nm}$ sputtered $\mathrm{Cr}$ layer serves as an adhesive coating between the quartz substrate and the $10 \mu \mathrm{m}$ thick sputtered Gd absorption layer, as shown in Fig. 2(a). The pattern transfer of the grating structure on the Gd layer, as illustrated in Fig. 2(b), is done by standard photolithography using a positive photoresist S1813 with a spin coated layer thickness of $1.3 \mu \mathrm{m}$. After a hard bake, the photoresist pattern serves as the etching mask for the Gd layer. The uncovered Gd is etched away in a diluted solution of sulfuric acid (96\% concentrated) and water with a mass ratio of $1: 100,{ }^{17}$ as depicted in Fig. 2(c). The resulting source grating is shown in Figs. 2(d) and 2(e), illustrating a section of a photograph of the processed wafer and a profilometer scan over two periods, respectively. The achieved height of about $11.5 \mu \mathrm{m}$, as measured with the profilometer, is composed of the $1.3 \mu \mathrm{m}$ resist layer and the sputtered $10.2 \mu \mathrm{m} \mathrm{Gd}$ absorption layer. The fabricated slit width $s=430 \mu \mathrm{m}$ for each line source and the Gd linewidth of $646 \mu \mathrm{m}$ give a DC of 0.4 for $G_{0}$.

The phase grating $G_{1}$ with a period of $7.97 \mu \mathrm{m}$, a Si structure height of $37 \mu \mathrm{m}$, and a DC of 0.5 is fabricated using a $100 \mathrm{~mm}\langle 110\rangle$ orientated silicon wafer as the substrate. ${ }^{18}$ The wafer is $300 \mu \mathrm{m}$ thick and polished on both sides. The wafer surface was thermally oxidized to give an $80 \mathrm{~nm}$ thin silicon dioxide $\left(\mathrm{SiO}_{2}\right)$ layer, which serves later as an etching mask for the silicon. The wafer size allows for a square grating size of $64 \times 64 \mathrm{~mm}^{2}$ in size. The fabrication processes are depicted on the top part of Fig. 3. In Fig. 3(a), the grating line pattern is transferred via optical photolithography into the spin coated $500 \mathrm{~nm}$ thick positive photoresist layer (S1805). The resist pattern is used as an etching mask to transfer the grid pattern into the $\mathrm{SiO}_{2}$ layer. A dry reactive ion etching process containing $\mathrm{CHF}_{3}$ and $\mathrm{O}_{2}$ as etching gases was used, as shown in Fig. 3(b). The remaining photoresist was stripped by an oxygen plasma etching step. To finally

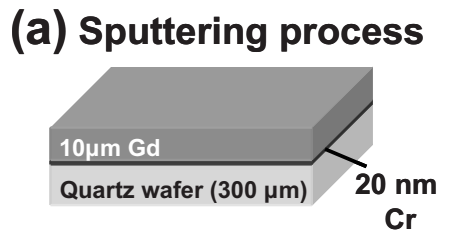

(d)



\section{(b) Photo lithography (c) Chemical wet etching}



(e)

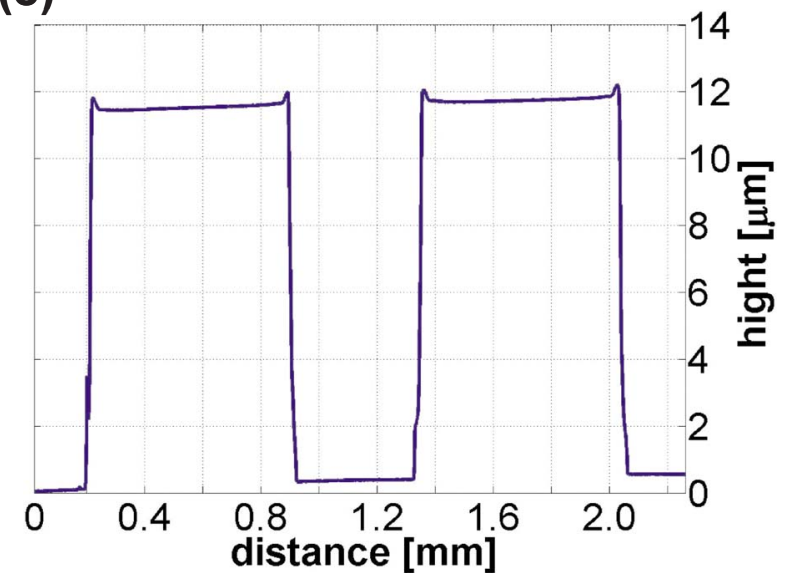

FIG. 2. (Color online) Fabrication of the source grating $G_{0}$. [(a)-(c)] Fabrication steps. (d) A photograph of a section of the processed wafer showing the Gd lines (black). (e) Profilometer scan over two grating periods denoting a structure height of $11.5 \mu \mathrm{m}$ consisting of $1.3 \mu \mathrm{m}$ resist layer and the $10.2 \mu \mathrm{m}$ Gd absorption layer with a DC of 0.4. 


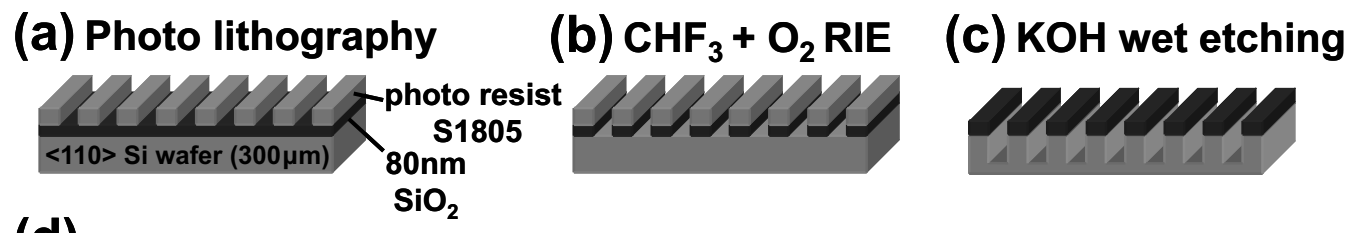

(d)

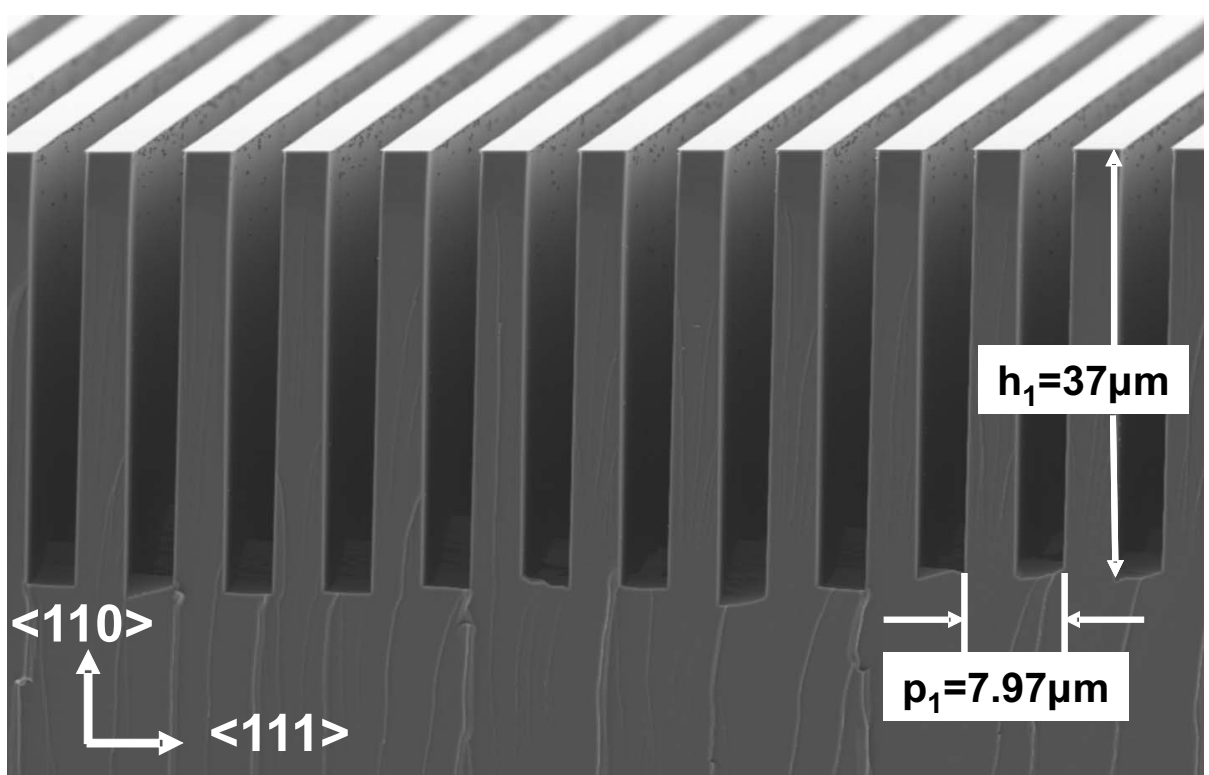

FIG. 3. Fabrication of the phase grating $G_{1}$. [(a)-(c)] Fabrication steps. (d) Cross section SEM image of the processed grating. The DC of 0.5 is properly achieved for the grating depth of $37 \mu \mathrm{m}$.

obtain the linear Si grating, the uncovered $\mathrm{Si}$ was etched in an anisotropic wet etching process in a $20 \%$ aqueous potassium hydroxide $(\mathrm{KOH})$ solution, as depicted in Fig. 3(c). The remaining $\mathrm{SiO}_{2}$ masking layer was removed in buffered oxide etch to achieve the final Si grating. A scanning electron microscope (SEM) cross section image of the processed wafer is shown in Fig. 3(d). Due to the fact that the etch rate of $1.68 \mu \mathrm{m} / \mathrm{min}$ at $76^{\circ} \mathrm{C}$ along the $\langle 110\rangle$ direction is 80 times faster than that along the $\langle 111\rangle$ direction, nearly perpendicu- lar sidewalls of the grating structures are achievable. As seen from the SEM image, the DC of 0.5 and the structure height of $37 \mu \mathrm{m}$ are achieved with high precision.

Manufacturing of the analyzer grating $G_{2}$ turned out to be the most challenging microfabrication task, as it has the smallest period of all three gratings, and it needs to consist of narrow Gd lines with sufficient height to absorb neutrons. Additionally, a large homogeneous grating area of 64 $\times 64 \mathrm{~mm}^{2}$ had to be fabricated to match the one of the phase (a)

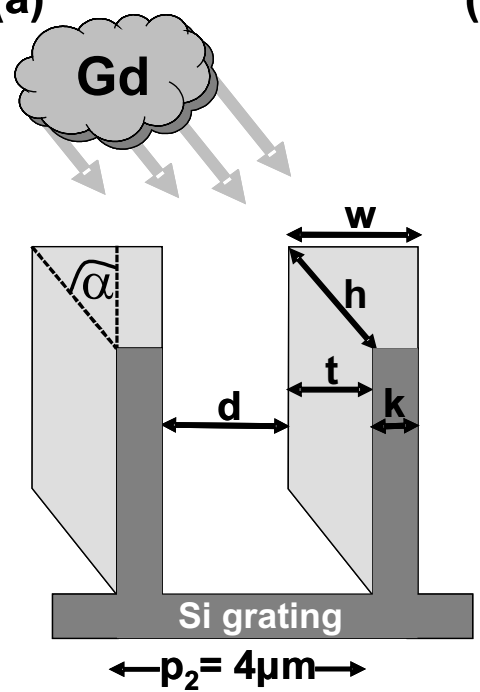

(b)

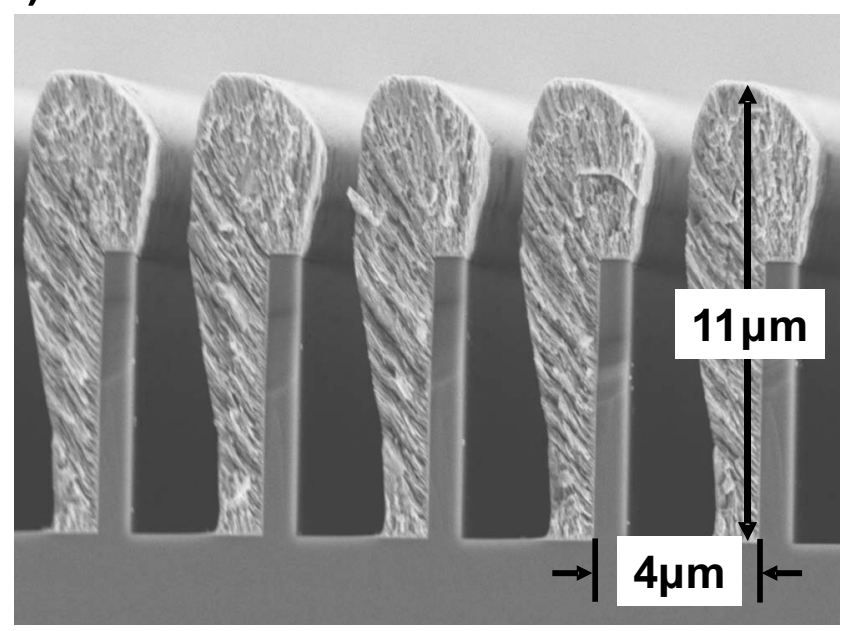

FIG. 4. Fabrication of the absorption grating $G_{2}$ by an inclined sidewall evaporation process. (a) Schematic view. (b) Cross section of a SEM image showing the $11 \mu \mathrm{m}$ high Gd structures with a DC of 0.25 . 
grating. The developed process for the analyzer grating includes an inclined sidewall evaporation process of $\mathrm{Gd}$ on a $\mathrm{Si}$ grating, as denoted in Fig. 4(a). Therefore, a Si grating with a period $p_{2}=4 \mu \mathrm{m}$ and a Si bar width $k$ that has to be as small as possible was fabricated on a $100 \mathrm{~mm}$ wafer with the same process steps as for the phase grating (Fig. 3). The fabrication limit for the smallest bar width $k$ was found to be $700 \mathrm{~nm}$ for a good grid line uniformity over the whole area. The produced Si grating serves as a basis for the inclined sidewall evaporation process. We used $\alpha=16^{\circ}$. The evaporated Gd height $h$ directly affects the thickness $t$ of the $\mathrm{Gd}$ at the sidewalls of the Si grating and, therefore, the DC of the final grating. From the geometric relations in Fig. 4(a), it follows that the $\mathrm{DC}$ is given by

$$
\mathrm{DC}=\frac{p_{2}-k-(h \sin \alpha)}{p_{2}} .
$$

For an evaporation height $h=3.7 \mu \mathrm{m}$, we obtain a DC of 0.5 . With the additional Gd layer on the sidewalls, the complete Gd structure height is $h_{2}=11 \mu \mathrm{m}$.

\section{CHARACTERIZATION OF THE GRATINGS}

The setup, as shown in Fig. 1(a), including the fabricated gratings was assembled at the ICON beamline ${ }^{12}$ at the spallation neutron source SINQ at PSI. The source size $c$ was defined by a circular aperture with a diameter of $20 \mathrm{~mm}$. The performance of the gratings was characterized by pixelwise evaluating the visibility $V \equiv\left(I_{\max }-I_{\min }\right) /\left(I_{\max }+I_{\min }\right)$, which is a quantity characterizing the produced contrast of the interferometer. When one of the gratings is scanned along the transverse direction $x_{g}$ [see Fig. 1(b)], the intensity signal $I\left(m, n, x_{g}\right)$ in each pixel $(m, n)$ oscillates as a function of $x_{g}$, as schematically shown for one pixel in Fig. 5(a). The measured neutron data reveal this oscillating behavior, as shown in Fig. 5(b). This intensity oscillation in each detector pixel can be expressed in a Fourier series,

$$
\begin{aligned}
I\left(m, n, x_{g}\right)= & \sum_{i=0} a_{i}(m, n) \cos \left[i k x_{g}+\phi_{i}(m, n)\right] \approx a_{0}(m, n) \\
& +a_{1}(m, n) \cos \left[k x_{g}+\phi_{1}(m, n)\right],
\end{aligned}
$$

where $a_{i}$ are the amplitude coefficients, $\phi_{i}$ are the corresponding phase coefficients, $k=2 \pi / p_{2}$, and $p_{2}$ is the period of the analyzer grating $G_{2}$.

The Fourier coefficients $a_{0}, a_{1}$, and $\phi_{1}$ appropriately describe the intensity oscillation, as the cosine function described by them fits well to the recorded intensity oscillation, as seen in Fig. 5(b). Therefore, the visibility in each detector pixel $V(m, n)$ can be quantified by the amplitude coefficients $a_{0}(m, n)$ and $a_{1}(m, n)$ to

$$
V(m, n) \equiv \frac{a_{1}(m, n)}{a_{0}(m, n)} .
$$

The result of the evaluated visibility over the whole grating area of $64 \times 64 \mathrm{~mm}^{2}$ for each detector pixel with the corresponding value of $V(m, n)$ is shown in Fig. 5(c). The visibility is homogeneously distributed over the grating area varying in the range from $21 \%$ to $25 \%$, with an average value of $23 \%$. It can be shown that the maximum achievable visibility (a)



(b)

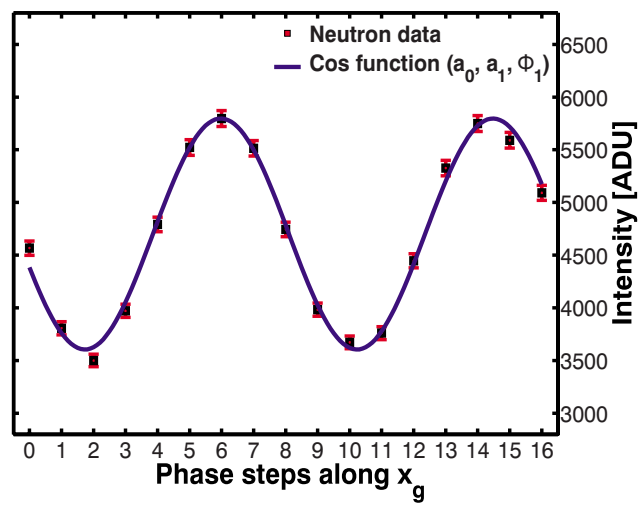

(c)

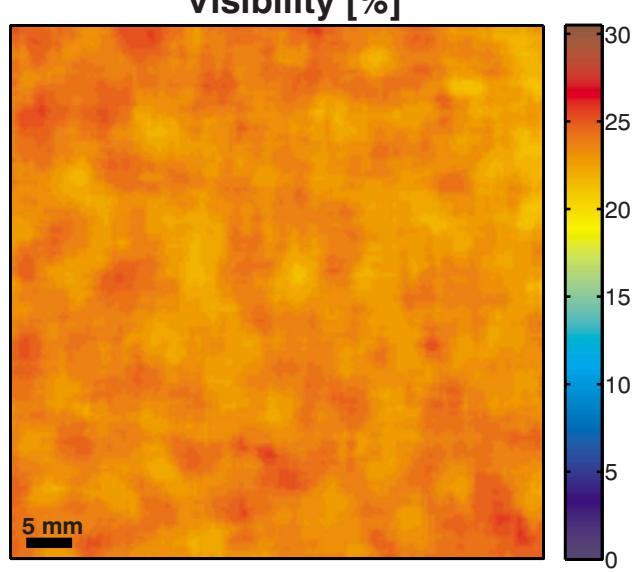

FIG. 5. (Color online) Characterization of the gratings. (a) Depiction of the intensity modulation for one detector pixel when one of the gratings is scanned along $x_{g}$ and the corresponding Fourier series coefficients $a_{0}, a_{1}$, and $\phi_{1}$ (see text). (b) Neutron data of the intensity modulation for a single detector pixel and the corresponding cosine function obtained from the extracted Fourier coefficients. (c) Visibility map over the whole grating area of $64 \times 64 \mathrm{~mm}^{2}$ showing the values of $a_{1} / a_{0}$ for each detector pixel with in an averaged value of $23 \%$.

for a three grating interferometer with perfectly opaque $G_{0}$ and $G_{2}$, an ideal phase grating $G_{1}$, and a DC of 0.5 for all gratings is $50 \%$ for a monochromatic beam. Taking into account the low temporal coherence conditions of our setup using a wavelength spectrum with a bandwidth of $\pm 10 \%$, delivered from a velocity selector, the achieved visibility is remarkably high.

For the evaluation of the visibility, 16 images with $10 \mathrm{~s}$ exposure time were taken over two periods of $G_{2}$. For the measurement, the DC of the source grating was set to 0.4, resulting in the largest slit width $s$ and maximum available flux. However, the largest value of $s$ leads to the smallest spatial coherence length of the setup resulting in the lowest visibility value. Therefore, reducing the slit width $s$ can additionally improve the visibility of the setup. 


\section{SUMMARY}

In summary, we have shown how diffraction gratings for neutron phase contrast imaging can be fabricated. On the one hand, the phase grating, which was fabricated from $\mathrm{Si}$, was fairly easy to produce since the used $\mathrm{KOH}$ wet etching technique is well known, even though the high precision concerning structure height and DC was quite challenging. On the other hand, two novel fabrication processes (due to different demands on the periodicity) for the Gd absorption gratings (source grating and analyzer grating) had to be developed since the deposition and patterning of $\mathrm{Gd}$ is uncommon in microfabrication technology. The fabricated gratings were characterized over the whole grating area by evaluating the measured contrast (visibility) in each detector pixel, resulting in an average value of $23 \%$. The produced grating size of $64 \times 64 \mathrm{~mm}^{2}$ was limited by the used $100 \mathrm{~mm}$ wafers. It can be scaled up to the nowadays standard $300 \mathrm{~mm}$ wafer size. This is an advantage compared to the other existing techniques regarding the maximum available sample size, in addition to the short data acquisition times with the grating interferometer setup.

\section{ACKNOWLEDGMENTS}

The authors gratefully acknowledge R. Bischofberger, A. Weber, F. Glaus, and B. Haas for their advice and help in context with the grating fabrication.
${ }^{1}$ H. Rauch, W. Treimer, and U. Bonse, Phys. Lett. 47A, 369 (1974).

${ }^{2}$ M. Zawisky, M. Baron, R. Loidl, and H. Rauch, Nucl. Instrum. Methods Phys. Res. A 481, 406 (2002).

${ }^{3}$ H. Rauch and S. A. Werner, Neutron Interferometry (Oxford University Press, Oxford, 2000).

${ }^{4}$ W. Treimer, M. Strobl, A. Hilger, C. Seifert, and U. Feye-Treimer, Appl. Phys. Lett. 83, 398 (2003).

${ }^{5}$ J. G. Barker, C. J. Glinka, J. J. Moyer, M. H. Kim, A. R. Drews, and M. Agamalian, J. Appl. Crystallogr. 38, 10041011 (2005).

${ }^{6}$ B. E. Allman, P. J. McMahon, K. A. Nugent, D. Paganin, D. L. Jacobson, and S. A. Werner, Nature (London) 408, 158 (2000).

${ }^{7}$ P. J. McMahon, B. E. Allman, M. Arif, S. A. Werner, and K. A. Nugent, Phys. Rev. Lett. 91, 145502 (2003).

${ }^{8}$ F. Pfeiffer, C. Grünzweig, O. Bunk, G. Frei, E. Lehmann, and C. David, Phys. Rev. Lett. 96, 215505 (2006)

${ }^{9}$ C. David, B. Nöhammer, E. Ziegler, and H. H. Solak, Appl. Phys. Lett. 81, 3287 (2002)

${ }^{10}$ T. Weitkamp, A. Diaz, F. Pfeiffer, M. Stampanoni, P. Cloetens, E. Ziegler, and C. David, Opt. Express 13, 6296 (2005).

${ }^{11}$ F. Pfeiffer, T. Weitkamp, O. Bunk, and C. David, Nat. Phys. 2, 258 (2006).

${ }^{12}$ G. Kühne, G. Frei, E. Lehmann, and P. Vontobel, Nucl. Instrum. Methods Phys. Res. A 542, 264 (2005).

${ }^{13}$ C. Grünzweig, Diploma thesis, University of Tübingen, 2006.

${ }^{14}$ C. Grünzweig, T. Hils, S. Mühlbauer, M. Ay, K. Lorenz, R. Georgii, R. Gähler, and P. Böni, Appl. Phys. Lett. 91, 203504 (2007).

${ }^{15}$ H. F. Talbot, Philos. Mag. 9, 401 (1836).

${ }^{16} \mathrm{http} / / /$ www.ncnr.nist.gov/resources/sldcalc.html

${ }^{17}$ C. Grünzweig, G. Frei, E. Lehmann, G. Kühne, and C. David, Rev. Sci. Instrum. 78, 053708 (2007).

${ }^{18}$ C. David, J. Bruder, T. Rohbeck, C. Grünzweig, C. Kottler, A. Diaz, O. Bunk, and F. Pfeiffer, Microelectron. Eng. 84, 1172 (2007). 